\title{
Estudio de las preocupaciones asociadas al embarazo en un grupo de embarazadas primigestas chilenas ${ }^{1}$
}

\section{Study of concerns related to pregnancy in a group of first-time Chilean pregnant women}

\author{
Chamarrita Farkas² y María Pía Santelices \\ Pontificia Universidad Católica de Chile
}

(Recepción: Enero 2008 - Aceptación: Mayo 2008)

\begin{abstract}
Resumen
Este artículo se centra en la identificación de las preocupaciones y temores que surgen en el período del primer embarazo. Se utiliza una metodología cualitativa, con un diseño transversal, exploratorio y descriptivo. La muestra se compone de 17 embarazadas primigestas de 19 a 40 años y nivel socio-económico medio, que asistieron a dos talleres de "Promoción del primer vínculo" con una frecuencia semanal durante 6 semanas. A partir de las transcripciones de las sesiones, se identificaron y construyeron categorías de respuesta asociadas a temores y preocupaciones de esta etapa. Los resultados se presentan considerando los temores y preocupaciones asociados al embarazo, al parto y a la primera etapa de la maternidad.
\end{abstract}

Palabras clave: Preocupaciones, Embarazadas primigestas

\begin{abstract}
This paper is focus in the identification of concerns and fears that emerges in the first pregnancy period. A qualitative methodology is used, with a transversal, exploratory and descriptive design. The sample was constituted by 17 first pregnancy women of 19 to 40 years old and medium socioeconomic level, whose assist to two workshops of "First bond promotion" with a weekly frequency during 6 weeks. From the transcription of the sessions, answers categories associates to concerns and fears in this stage were identify and built. The results are presented considering the concerns and fears associate to the pregnancy, to the childbirth and to the early motherhood.
\end{abstract}

Key words: Concerns, First pregnancy

El embarazo forma parte natural de la vida de la mayoría de las mujeres, y constituye una tarea reproductiva y evolutiva, que gatilla un proceso de redefinición en la mujer en el cual ésta pasa a convertirse en madre (Oiberman, 2001). Es un período dinámico, de gran permeabilidad y vulnerabilidad, de mayor sensibilidad a las interacciones, acompañado de inestabilidad emocional y sentimientos ambivalentes (Gauthier, Molenat, Mangin \& Dudan, 1985; Sevon, 2005).

Las principales tareas psicológicas para la mujer son el logro de una fusión emocional con el bebé intrauterino en períodos tempranos del embarazo, luego diferenciarse gradualmente y finalmente una progresiva separación psíquica entre ella y su bebé, la cual culmina en la separación física durante el nacimiento (Raphael-Leff, 1982). En esta etapa la mujer percibe la pérdida del control de su cuerpo, lo cual suele generar inquietud y angustia (Warren \& Brewis, 2004).

1 La elaboración de este artículo contó con el financiamiento otorgado por el Fondo Nacional de Desarrollo Científico y Tecnológico, FONDECYT, No 1040760.

2 Correspondencia a: Chamarrita Farkas. Escuela de Psicología, Pontificia Universidad Católica de Chile. Dirección: Escuela de Psicología Pontificia Universidad Católica de Chile, Avda. Vicuña Mackenna 4860, Macul Santiago, CHILE Teléfono: 56-2-3547067 Fax: 56-2-3544844 E-mail: chfarkas@uc.cl 


\section{Preparación Psicológica para el Parto y la Maternidad Temprana}

El parto constituye el proceso a través del cual el embarazo llega a su término y nace el bebé, separándose físicamente de su madre. Es un proceso no falto de riesgos y durante el embarazo la mujer debe prepararse física y psicológicamente para este momento. Constituye un "período sensible” y lo que ocurra durante éste determinará de manera importante su relación afectiva con el bebé, la calidad de los cuidados, su autoestima, bienestar, incluso su relación de pareja (Valdés \& Morlans, 2005).

Luego del parto la mujer experimentará una serie de cambios hormonales y biológicos, traduciéndose en una mayor vulnerabilidad psicológica y susceptibilidad a desarrollar una disforia o depresión postnatal. Deberá aprender a anteponer las necesidades del bebé (ej., lactancia) a las propias, y aceptar los cambios corporales que se producen (Patel, Lee, Wheatcroft, Barnes \& Stein, 2005). Deberá recuperarse física y psicológicamente del parto, establecer sentimientos hacia su hijo, desarrollar la identidad de madre y nuevas maneras de relacionarse (Woollett \& Parr, 1997).

En esta etapa son fundamentales ciertos recursos de la madre, por ejemplo la sensibilidad materna, que la ayudarán a percibir y responder de manera adecuada a las necesidades de su bebé (Stern, 1997). Así, se han descrito a través de investigaciones factores que afectan negativamente la parentalidad, como el estrés y la baja sensibilidad, que no permiten la capacidad reguladora por parte de la madre del estado emocional de sus bebés (Cerezo, Trenado \& Pons-Salvador, 2006), afectando la interacción temprana que se establece desde el inicio entre ambos.

\section{El Primer Embarazo}

El primer embarazo es de gran significancia, ya que en éste se produce la preparación para la maternidad. La forma en que las tareas del embarazo se manejen, será predictiva de la adaptación al rol maternal y del crecimiento psicológico que se experimente durante el embarazo y parentalidad temprana (Leifer, 1977).

Se produce además un importante cambio en el estilo de vida, pudiendo conllevar a sentimientos ambivalentes y negativos hacia el bebé, lo cual no ocurre de igual forma en embarazos posteriores (Smith, 1999). Un estudio realizado en Estados Unidos con 701 embarazadas, comprobó que las primigestas presentaban un mayor porcentaje de embarazos no planificados, lo cual incrementa la posibilidad de tener sentimientos ambivalentes y negativos hacia el bebé (Speitzer, Santelli, AfableMunsuz \& Kendall, 2004).

\section{Actitudes y Preocupaciones de la Mujer hacia el Embarazo}

En las actitudes de la mujer frente al embarazo, cabe considerar sus expectativas y sentimientos hacia su bebé y su rol de futura madre, y si es un embarazo deseado. Actitudes negativas hacia el embarazo aumentan el estrés de la experiencia y las consecuencias psicológicas adversas, mientras que una actitud positiva disminuye el estrés y la ansiedad, y protegen a la mujer de posibles consecuencias psicológicas (Gurung, Dunkel-Schetter, Collins, Rini \& Hobel, 2005; Zambrana, Scrimshaw, Collins \& Dunkel-Schetter, 1997).

Al estudiar las preocupaciones y actitudes de las mujeres hacia sus embarazos se han encontrado relaciones entre éstas, y el posterior desarrollo del bebé. Un estudio realizado en Inglaterra con 436 embarazadas primigestas, correlacionó una actitud más positiva hacia el embarazo con mayor conciencia de los cambios que implicaba la maternidad e hijos con un mejor desarrollo cognitivo a los 2 años de edad (Deave, 2005).

Una investigación realizada en Inglaterra con 1.207 embarazadas, reporta preocupaciones relacionadas con la propia salud y con aspectos socio-médicos, socioeconómicos y relacionales (Green, Kafetsios, Statham \& Snowdon, 2003). 
Existe una importante preocupación en las mujeres después del parto por su apariencia corporal y peso, correlacionándose ello con su autoestima e identidad (Woollett \& Parr, 1997). En un estudio en EEUU sobre las preocupaciones del embarazo, un 94\% mencionó la ganancia de peso durante el embarazo, un 44\% refirió como preocupación la salud de sus bebés, y un 26,2\% lo hizo respecto a su apariencia después del embarazo (Obesity, Fitness \& Wellness Week Editors, 2004). Esta preocupación es comprensible debido al acento cultural en la apariencia física de las mujeres (Jordan, Capdevila \& Johnson, 2005).

Otra preocupación común es el nacimiento del niño. Incluso ha sido descrito como el "fenómeno del miedo al nacimiento del bebé" (fear of childbirth phenomenon, FOC). Este temor puede aparecer durante el embarazo, el parto, o incluso durante el período del post-parto. Las mujeres que lo experimentan durante el embarazo, suelen tener niveles de miedo más intensos durante el parto y pueden desarrollar incluso síntomas correspondientes a un desorden de estrés post-traumático (Wijma, 2003).

Estudios en mujeres que habían experimentado pérdidas anteriores identificaron preocupaciones relacionadas con perder otro bebé, la salud general de éste, la estabilidad emocional propia, el impacto de otra pérdida en el futuro, la falta de apoyo de otras personas y el miedo a recibir malas noticias (Côte-Arsenault \& Bidlack, 2001). Respecto a las preocupaciones en mujeres sobre los 35 años de edad, éstas se refieren principalmente al bienestar del bebé intrauterino y a temas de salud de la madre (Viau, 2002).

El objetivo del presente artículo es identificar y describir las preocupaciones de un grupo de embarazadas primigestas en Chile. La aproximación a la compleja experiencia del embarazo y la comprensión de las preocupaciones en esta etapa ayudará a los profesionales de la salud a entregar un adecuado apoyo a las mujeres, incidiendo positivamente en el desarrollo futuro de la madre y del bebé.

\section{Método}

\section{Diseño}

Este estudio optó por un diseño metodológico cualitativo, transversal, exploratorio y descriptivo, privilegiando una aproximación a las cualidades del objeto de estudio (Strauss \& Corbin, 1990). Se describen las preocupaciones de un grupo de embarazadas primigestas, a partir de sus discursos durante su participación en talleres grupales.

\section{Participantes}

Las participantes fueron 17 embarazadas primigestas entre 20 y 34 años, de nivel socioeconómico medio, participantes de dos talleres "Promoción de Apego Seguro" en un centro médico (8 y 9 participantes). Los talleres consideraron 6 sesiones de frecuencia semanal, de dos horas cada una. Los criterios de inclusión en la muestra eran la edad y ser el primer embarazo. Como exclusión se consideró la presencia de diagnóstico psiquiátrico grave.

\section{Procedimiento}

Las participantes fueron contactadas en un centro médico. La participación fue voluntaria, confidencial y se firmó una carta de consentimiento. Los talleres tuvieron como objetivo promover un vínculo sano con los futuros bebés, incluyendo aspectos educativos y de apoyo afectivo. Fueron realizados por dos psicólogas especialistas en el tema ${ }^{3}$. Todas las sesiones fueron filmadas y transcritas.

3 Estos talleres forman parte del Proyecto FONDECYT N 1040760 "Promoción de apego seguro: Evaluación de un programa de intervención en futuras madres primigestas”. 
Instrumento de recolección de datos

La recolección de datos se realizó a través de la filmación de las sesiones, las cuales luego fueron transcritas en su cabalidad.

Para el análisis se identificaron segmentos del discurso que expresaran preocupaciones, considerando que éstas:

- surgieran espontáneamente

- se manifestaran en sesión

- fueran coherentes y consistentes

- fueran referidas por las participantes como una preocupación.

A partir de la información recolectada se construyeron categorías de respuesta por medio de un proceso inductivo, desde las narrativas de las participantes (Valles, 1999).

\section{Resultados}

Se recogió un total de 66 preocupaciones que se agruparon en tres categorías, que a su vez se dividieron en 5 subcategorías cada una. Las categorías principales son: Temores y preocupaciones asociadas al embarazo (27\%), asociadas al parto (17\%) y asociadas a la maternidad temprana (56\%). Las subcategorías se describen según mayor a menor frecuencia de aparición (Tabla 1).

Tabla 1. Distribución de frecuencia de temores y preocupaciones detectados

\begin{tabular}{|c|c|c|c|}
\hline Categoría & Subcategoría & $\begin{array}{l}\text { Frecuencia } \\
\text { Respecto al total }\end{array}$ & $\begin{array}{l}\text { Frecuencia } \\
\text { intracategoría }\end{array}$ \\
\hline \multirow{6}{*}{$\begin{array}{l}\text { Temores y } \\
\text { preocupaciones asociadas } \\
\text { a la etapa del embarazo }\end{array}$} & Preocupación de perder al bebé & $9 \%$ & $33 \%$ \\
\hline & $\begin{array}{l}\text { Preocupación frente a problemas del bebé por } \\
\text { problemas en el embarazo }\end{array}$ & $6 \%$ & $22 \%$ \\
\hline & Preocupación frente a lo desconocido & $6 \%$ & $22 \%$ \\
\hline & $\begin{array}{l}\text { Preocupación frente a problemas del bebé relacionados } \\
\text { a características de la madre }\end{array}$ & $3 \%$ & $11 \%$ \\
\hline & Preocupación de dañar al bebé & $3 \%$ & $11 \%$ \\
\hline & FRECUENCIA CATEGORÍA & $27 \%$ & $100 \%$ \\
\hline \multirow{6}{*}{$\begin{array}{l}\text { Temores y } \\
\text { preocupaciones asociadas } \\
\text { al parto }\end{array}$} & Preocupación frente al dolor del parto & $6 \%$ & $35 \%$ \\
\hline & $\begin{array}{l}\text { Preocupación de no poder identificar el inicio del } \\
\text { trabajo de parto }\end{array}$ & $3 \%$ & $17 \%$ \\
\hline & Preocupación por la reacción propia o de otras personas & $3 \%$ & $17 \%$ \\
\hline & Preocupación por perder el control de la situación & $3 \%$ & $17 \%$ \\
\hline & Preocupación frente a lo desconocido del parto & $2 \%$ & $12 \%$ \\
\hline & FRECUENCIA CATEGORÍA & $17 \%$ & $100 \%$ \\
\hline \multirow{6}{*}{$\begin{array}{l}\text { Temores y } \\
\text { preocupaciones asociadas } \\
\text { a la etapa de maternidad } \\
\text { temprana }\end{array}$} & Preocupación frente al rol de la maternidad & $29 \%$ & $52 \%$ \\
\hline & Preocupaciones frente a aspectos del bebé & $11 \%$ & $20 \%$ \\
\hline & Preocupaciones asociadas a aspectos personales & $9 \%$ & $16 \%$ \\
\hline & Preocupaciones frente a aspectos relacionales & $4 \%$ & $7 \%$ \\
\hline & Preocupaciones frente a aspectos contextuales & $3 \%$ & $5 \%$ \\
\hline & FRECUENCIA CATEGORÍA & $56 \%$ & $100 \%$ \\
\hline
\end{tabular}




\section{Temores y Preocupaciones Asociadas a la Etapa del Embarazo}

Estas preocupaciones se centran en los cambios experimentados durante el embarazo y consideran 5 subcategorías. La primera se refiere a la preocupación a perder al bebé, (33\%) ya sea por experiencias previas de pérdida o sin ellas. La segunda considera la preocupación frente a problemas del bebé por problemas en el embarazo (22\%). Este temor se refiere a que problemas del embarazo puedan afectar al bebé, de manera física o psicológica.

La tercera es la preocupación frente a lo desconocido (22\%). Ésta se relaciona con la experiencia del primer embarazo, y se suscita frente a sensaciones desconocidas hasta ahora, así como frente a la falta de información. Al respecto, aparecen expresiones de ansiedad o temor generalizado frente a esta nueva etapa y lo que ella implica, incluida la pérdida de control sobre la propia vida. También se encuentra la preocupación frente a problemas del bebé relacionados a características de la madre (11\%). Considera características propias de la madre que afecten físicamente el desarrollo del bebé como su edad, o características más situacionales como estar deprimida o con problemas de pareja.

Finalmente, se aprecia la preocupación de dañar al bebé (11\%). Esta alude a conductas que realiza la mujer durante su embarazo y que puedan dañar al bebé, como manejar, hacer demasiadas cosas o caerse.

\section{Temores y Preocupaciones Asociadas al Parto}

Estas preocupaciones se refieren al momento del parto, identificándose 5 subcategorías. La primera se refiere a la preocupación frente al dolor del parto (35\%). Esta preocupación se refiere al dolor físico que se sentirá antes y durante el parto, y cómo manejarlo. Una segunda preocupación es el no poder identificar el inicio del trabajo de parto (17\%).

Otra preocupación se refiere a la reacción propia o de otras personas (17\%), la cual alude a las preocupaciones relacionadas con la reacción que se tendrá en la situación del parto, ya sea la propia reacción a la situación, como la reacción de otras personas cercanas como la pareja y su familia

También aparece la preocupación por perder el control de la situación (17\%). Ello se relaciona con que otros se hagan cargo, y no uno mismo. Se asocia a sentimientos de impotencia, y un deseo de las embarazadas de poder controlar la situación como una forma de manejar la angustia. Finalmente, aparece la preocupación a lo desconocido del parto (12\%), la cual guarda relación con no saber a qué se enfrentan, y la sensación de no saber como actuar cuando llegue el momento.

\section{Temores y Preocupaciones Asociadas a la Etapa de Maternidad Temprana}

Estas preocupaciones reflejan la anticipación y la preparación que ya se inicia frente a los cambios radicales que implicará la llegada del bebé y corresponden a la categoría de mayor frecuencia $(56 \%$ del total). Las preocupaciones se dan principalmente respecto a no poder asumir correctamente el rol de la maternidad, pero además se manifiestan frente a aspectos personales, del bebé, relacionales y contextuales.

Preocupaciones frente al rol de la maternidad (52\%). Una preocupación compartida por las mujeres durante los talleres, fue el no poder cumplir con lo que ellas esperaban -o su cultura-respecto a "ser una buena madre". Estas preocupaciones se daban frente a temas específicos como el no lograr ser una buena madre, no lograr entender las necesidades del bebé o no estar lo suficientemente atenta a lo que le pasa, no poder amamantarlo, sentir rechazo hacia él o no ser capaz de hacerlo bien en caso de déficit, traspasarle sus propios problemas o que a futuro el hijo le reclame por no haberlo hecho bien.

Preocupaciones frente a aspectos del bebé (20\%). En esta categoría aparecen preocupaciones frente a cómo será el bebé, ya sea que sus expectativas no se cumplan, que el bebé no sea sano, que presente características negativas o anomalías no detectadas previamente. Esta última preocupación se refiere a encontrarse en el nacimiento con anomalías de su bebé, no detectadas durante el embarazo, 
lo cual también se relaciona con frustración de expectativas previas. Se da en mujeres que han vivido situaciones de este tipo en casos cercanos, les genera mucha angustia y suelen desconfiar de los equipos médicos y de los exámenes.

Preocupaciones asociadas a aspectos personales (16\%). Esta subcategoría se refiere a las preocupaciones que se sienten frente a los cambios en la vida personal que la maternidad generará, algunos de ellos temporales y otros más estables. Los aspectos personales mencionados son cambios afectivos (principalmente desórdenes hormonales y depresión postparto), cambios laborales o económicos, cambios en el tiempo libre, cambios en las rutinas y problemas para compatibilizar el rol de madre con el de mujer.

Preocupaciones frente a aspectos relacionales (7\%). Se refiere a preocupaciones sobre la relación que se establecerá con el propio bebé, especialmente por la dependencia que ello implica, y cómo esta nueva relación afectará las relaciones con otras personas cercanas, en especial la pareja y la familia de origen, e incluso una mascota.

Preocupaciones frente a aspectos contextuales (5\%). Finalmente aparecen preocupaciones frente al contexto, y cómo esto puede afectar al bebé. Esta preocupación aparece en las embarazadas solteras, en relación al efecto negativo que pueda tener la ausencia del padre.

\section{Discusión}

Al explorar las preocupaciones y temores de un grupo de embarazadas primigestas, las expresiones más frecuentes se relacionaron con la etapa de la maternidad temprana. Ello puede entenderse por el carácter preparatorio que tiene el embarazo frente a la futura maternidad y los temores de enfrentar el vínculo con el bebé, una vez nacido.

Un segundo ámbito de preocupaciones se refiere al proceso experimentado durante el embarazo, el cual alude a temores y ansiedades frente al adecuado desarrollo del bebé y su sobrevida. Finalmente, el momento del parto también genera ansiedad, ya que es una experiencia desconocida asociada a dolor físico y que genera intensos sentimientos de pérdida del control de la situación.

Al comparar este estudio con otras investigaciones, igualmente aparecen preocupaciones relacionadas con el parto, temores frente a situaciones de pérdidas anteriores y la influencia de ciertos aspectos como por ejemplo una edad más avanzada (Côte-Arsenault \& Bidlack, 2001; Viau, 2002; Wijma, 2003). En cambio no se manifiesta la preocupación por el peso y el cambio en las formas corporales, como muestran otros estudios (Patel et al., 2005), lo cual podría atribuirse a las características metodológicas del estudio o a diferencias culturales.

La mayoría de las preocupaciones expresadas en esta investigación reflejan sentimientos ambivalentes que son esperables en este período. A su vez, es importante para los equipos de salud estar atentos a la expresión de ambivalencia excesiva, la cual es indicativa de dificultades actuales o futuras, expresándose en emociones excesivamente negativas en relación al embarazo o al bebé, la incapacidad de imaginarse al bebé, la exacerbación de las molestias físicas, y deseos inconscientes de dañar al bebé, los cuales se manifiestan en temores infundados de accidentes o situaciones catastróficas. Estas dificultades pueden indicar una depresión preparto, la cual podría relacionarse con una futura depresión postparto, afectando a la madre y al bebé.

La relevancia de la detección y prevención de problemas psicológicos durante la atención primaria de salud, ya ha sido resaltada por otros estudios (ver por ejemplo estudio de González, Fernández, Pérez \& Amigo, 2006). Ello se hace aun más evidente en el caso del control de salud de las embarazadas, por la posibilidad de intervenir oportunamente tanto en la salud mental de la madre como en el desarrollo de un vínculo sano con el bebé. Al dar un espacio de escucha a las preocupaciones de las embarazadas, se abre un importante campo de prevención primaria, en el sentido de poder detectar precozmente cómo este proceso se está desarrollando tanto a nivel físico como emocional, y poder identificar aquellas preocupaciones indicativas de problemas presentes y 
futuros.

En síntesis, el presente estudio muestra que las preocupaciones de este grupo de embarazadas chilenas se focaliza en las tareas que implica el embarazo, entre ellas el prepararse adecuadamente para una nueva identidad, y el proceso de convertirse en madre. El conocer como estas preocupaciones se manifiestan en nuestra población puede ayudar a los equipos de salud, desde una mirada interdisciplinaria, a apoyar a la mujer, aclarando y acogiendo sus dudas, poniendo atención frente a sus preocupaciones, y en caso de ser necesario, derivarla oportunamente a especialistas en salud mental.

\section{Referencias}

Cerezo, M. A., Trenado, R. M. \& Pons-Salvador, G. (2006). Interacción temprana madre-hijo y factores que afectan negativamente la parentalidad. Psicothema, 18, 3, 544-550.

Côte-Arsenault, D. \& Bidlack, D. (2001). Women's emotions and concerns during pregnancy following perinatal loss. MCN: The American Journal of Maternal/Child Nursing, 26, 3, 128 134.

Deave, T. (2005). Associations between child development and women's attitudes to pregnancy and motherhood. Journal of Reproductive and Infant Psychology, 23, 1, 63.

Gauthier, Y., Molenat, F., Mangin, P. \& Dudan, E. (1985). Grossesse et vulnerabilité. Neuropsychiatrie de l'Enfance et de l'Adolescence. 33 (2-3), 95-102.

Green, J. M., Kafetsios, K., Statham, H. M. \& Snowdon, C. M. (2003). Factor structure, validity and reliability of the Cambridge Worry Scale in a pregnant population. Journal of Health Psychology, $8,6,753-764$.

González, S., Fernández, C., Pérez, J. \& Amigo, I. (2006). Prevención secundaria de la depresión en atención primaria. Psicothema, 18, 3, 471-477.

Gurung, R. A., Dunkel-Schetter, C., Collins, N., Rini, C. \& Hobel, C. J. (2005). Psychosocial predictors of prenatal anxiety. Journal of Social and Clinical Psychology, 24, 4, 497-519.

Jordan, K., Capdevila, R. \& Johnson, S. (2005). Baby or beauty: A Q study into post pregnancy body image. Journal of Reproductive and Infant Psychology, 23, 1, 19-31.

Leifer, M. (1977). Psychological changes accompanying pregnancy and motherhood. Genetic Psychology Monographs, 95, 1, 55-96.

Obesity, Fitness \& Wellness Week Editors (2004, Nov 27). Nutrition; most pregnant women concerned about weight gain during pregnancy. Obesity, Fitness \& Wellness Week, p. 1009.

Oiberman, A. (2001). Observando a los bebés. Buenos Aires, Argentina: Lugar.

Patel, P., Lee, J., Wheatcroft, R., Barnes, J. \& Stein, A. (2005). Concerns about body shape and weight in the postpartum period and their relation to women's self-identification. Journal of Reproductive and Infant Psychology, 23, 4, 347-364.

Raphael-Leff, J. (1982). Psychotherapeutic needs of mothers-to-be. Journal of Child Psychotherapy, $8,1,3-13$.

Sevon, E. (2005). Timing motherhood: Experiencing and narrating the choice to become a mother. Feminism \& Psychology, 15, 4, 461.

Stern, D. (1997). La constelación maternal. Un enfoque unificado de la psicoterapia con padres e hijos. Madrid, España: Paidós.

Smith, J. (1999). Towards a relational self: Social engagement during pregnancy and psychological preparation for motherhood. The British Journal of Social Psychology, 38, 4, 409-428. 
Speitzer, I., Santelli, J., Afable-Munsuz, A. \& Kendall, C. (2004). Measuring factors underlying intendedness of women's first and later pregnancies. Perspectives on Sexual and Reproductive Health, 36, 5, 198-205.

Strauss, A. \& Corbin, J. (1990). Basic of qualitative research: Grounded theory procedures and techniques. London: Sage.

Valdés, V. \& Morlans, X. (2005). Aportes de las doulas a la obstetricia moderna. Revista Chilena de Obstetricia y Ginecología, 70, 2, 108-112.

Valles, M. (1999). Técnicas cualitativas de investigación social: Reflexión metodológica y práctica profesional. Madrid: Síntesis Sociológica.

Viau, P. (2002). An exploration of health concerns \& health-promotion behaviors in pregnant women over age 35. MCN: The American Journal of Maternal/Child Nursing, 27, 6, 328-334.

Warren, S. \& Brewis, J. (2004). Matter over mind? Examining the experience of pregnancy. Sociology: The Journal of the British Sociological Association, 38, 2, 219.

Wijma, K. (2003). Why focus on "fear of childbirth”? Journal of Psychosomatic Obstetrics \& Gynecology, 24, 3, 141-143.

Woollett, A. \& Parr, M. (1997). Psychological tasks for women and men in the post-partum. Journal of Reproductive and Infant Psychology, 15, 2, 159-183.

Zambrana, R. E., Scrimshaw, S. C., Collins, N. \& Dunkel-Schetter, C. (1997). Prenatal health behaviors and psychosocial risk factors in pregnant women of Mexican origin: The role of acculturation. American Journal of Public Health, 87, 1022-1026. 